\title{
SOME PROPERTIES OF MEROMORPHIC FUNCTIONS CONCERNING SHARED-SETS
}

\author{
AI-Di WU AND WEI-CHUAN LIN
}

\begin{abstract}
Using Nevanlinna's value distribution theory, we study shared-set problems of meromorphic functions and prove that there exist three finite sets $S_{1}\left(\sharp\left(S_{1}\right)=1\right), S_{2} \quad\left(\sharp\left(S_{2}\right)=1\right)$ and $S_{3}\left(\sharp\left(S_{3}\right)=5\right)$ such that any two meromorphic functions $f$ and $g$ sharing $S_{j}(j=1,2,3)$ must be identical. Our results are improvements of those of former authors and the complement of Ref. [W. Lin and H. X. Yi, Uniqueness theorems for meromorphic functions that share three sets. Complex Variables, 44 (2003), 315-327.]. In addition, we show the accuracy of the results by giving some examples.
\end{abstract}

Mathematics subject classification (2010): 30D30, 30D35.

Keywords and phrases: Meromorphic function, sharing set, uniqueness.

\section{REFERENCES}

[1] T. B. CAO, K. LIU AND N. XU, Zeros and uniqueness of q-difference polynomials of meromorphic functions with zero order, Proc. Indian Acad. Sci., 124 (4) (2014), 533-549.

[2] M. L. FAng AND H. Guo, On meromorphic functions sharing two values, Analysis, 17 (1997), 355 366.

[3] M. L. FANG AND I. LAHIRI, Unique range set for certain meromorphic functions, Indian J. Math., 45 (2) (2003), 141-150.

[4] G. FRANK AND M. ReINDERS, A unique range set for meromorphic functions with 11 elements, Complex Variables, 37 (1998), 185-193.

[5] H. Fujimoto, On uniqueness of meromorphic functions sharing finite sets, Amer. J. Math., 122 (2000), 1175-1203.

[6] F. Gross, Factorization of meromorphic functions and some problems, Complex analysis (Proc. Conf., Univ. Kentucky, Lexington, Ky, 1976), 51-76, Lecture Notes in Math. 599, Springer-Verlag, Berlin, 1977.

[7] W. K. Hayman, Meromorphic Functions, Claredon Press, Oxford, 1964.

[8] I. LAHIRI, The range set of meromorphic derivatives, Northeast. Math. J., 14 (3) (1998), 353-360.

[9] P. Li AND C. C. YANG, On the unique range set of meromorphic functions, Proc. of Amer. Math. Soc., 124 (1996), 177-185.

[10] W. C. LIN AND H. X. YI, Uniqueness theorems for meromorphic functions that share three sets, Complex Variables, 44 (2003), 315-327.

[11] V. N. Mishra, Some Problems on Approximations of Functions in Banach Spaces, Ph. D. Thesis (2007), Indian Institute of Technology, Roorkee - 247667 , Uttarakhand, India.

[12] E. MUES AND M. REINDERS, Meromorphic functions sharing one value and unique range sets, Kodai Math. J., 18 (1995), 515-522.

[13] R. Nevanlinna, Le Théorème de Picard-Borel et la Théorie des Fonctions Meromorphes, GauthiersVillars, Paris, 1929.

[14] L. YANG, Value Distribution Theory, Springer-Verlag, Berlin, 1993.

[15] B. YI AND Y. H. LI, The uniqueness of meromorphic functions that share two sets with CM, Acta. Math. Sin, Chinese Series, (2) 55 (2012), 363-368.

[16] H. X. Yi AND C. C. YANG, Uniqueness Theory of Meromorphic Functions, Science Press, Beijing, 1995. 
[17] H. X. YI, Uniqueness of meromorphic functions and a question of Gross, Science in China, Sr. A, (5) 24 (1994), 457-466.

[18] H. X. Y I, Unicity theorems for meromorphic or entire functions II, Bull. Austral. Math. Soc, 52 (1995), 215-224.

[19] H. X. YI, On a question of Gross concering uniqueness of entire functions, Bull. Austral. Math. Soc. 57 (1998), 343-349.

[20] H. X. YI, Meromorphic functions that share two sets, Acta Mathematica Sinica, Chinese Scies, (5) 45 (2002), 75-82.

[21] H. X. YI, Meromorphic functions that share one or two values, Complex Variables, 28 (1995), 1-11.

[22] H. X. YI AND W. C. LIN, Uniqueness of meromorphic functions and a question of Gross, Kyungpook Math. J, 46 (2006), 437-444. 